\title{
Chronic co-implantation of ultraflexible neural electrodes and a cranial window
}

\author{
Rongkang Yin,,${ }^{a, b, \dagger}$ Brian C. Noble, ${ }^{b, c, \dagger}$ Fei He,,${ }^{\text {a,b }}$ Pavlo Zolotavin,, a,b \\ Haad Rathore $\odot,{ }^{b, c}$ Yifu Jin, ${ }^{\text {a,b }}$ Nicole Sevilla,, ${ }^{\text {b,d }}$ Chong Xie, ${ }^{\text {a,b,d }}$ \\ and Lan Luan $\oplus^{\mathbf{a}, \mathbf{b}, \mathbf{d}, *}$ \\ ${ }^{a}$ Rice University, Department of Electrical and Computer Engineering, Houston, \\ Texas, United States \\ ${ }^{\mathrm{b}}$ Rice University, Rice Neuroengineering Initiative, Houston, Texas, United States \\ ${ }^{\mathrm{c}}$ Rice University, Applied Physics Graduate Program, Houston, Texas, United States \\ ${ }^{\mathrm{d}}$ Rice University, Department of Bioengineering, Houston, Texas, United States
}

\begin{abstract}
Significance: Electrophysiological recording and optical imaging are two prevalent neurotechnologies with complementary strengths, the combined application of which can significantly improve our capacity in deciphering neural circuits. Flexible electrode arrays can support longitudinal optical imaging in the same brain region, but their mechanical flexibility makes surgical preparation challenging. Here, we provide a step-by-step protocol by which an ultraflexible nanoelectronic thread is co-implanted with a cranial window in a single surgery to enable chronic, dual-modal measurements.
\end{abstract}

Aim: The method uses 1- $\mu$ m-thick polymer neural electrodes which conform to the site of implantation. The mechanical flexibility of the probe allows bending without breaking and enables long-lasting electrophysiological recordings of single-unit activities and concurrent, high-resolution optical imaging through the cranial window.

Approach: The protocol describes methods and procedures to co-implant an ultraflexible electrode array and a glass cranial window in the mouse neocortex. The implantation strategy includes temporary attachment of flexible electrodes to a retractable tungsten-microwire insertion shuttle, craniotomy, stereotaxic insertion of the electrode array, skull fixation of the cranial window and electrode, and installation of a head plate.

Results: The resultant implant allows simultaneous interrogation of brain activity both electrophysiologically and optically for several months. Importantly, a variety of optical imaging modalities, including wide-field fluorescent imaging, two-photon microscopy, and functional optical imaging, can be readily applied to the specific brain region where ultraflexible electrodes record from.

Conclusions: The protocol describes a method for co-implantation of ultraflexible neural electrodes and a cranial window for chronic, multimodal measurements of brain activity in mice. Device preparation and surgical implantation are described in detail to guide the adaptation of these methods for other flexible neural implants and cranial windows.

(C) The Authors. Published by SPIE under a Creative Commons Attribution 4.0 International License. Distribution or reproduction of this work in whole or in part requires full attribution of the original publication, including its DOI. [DOI: 10.1117/1.NPh.9.3.032204]

Keywords: flexible electrodes; cranial window; electrophysiology; optical imaging.

Paper 21045PRTSSR received Sep. 6, 2021; accepted for publication Nov. 4, 2021; published online Jan. 7, 2022.

\section{Introduction}

The brain has an enormous dynamic range spatially and temporally. Temporally, moment-bymoment information is processed at milliseconds, ${ }^{1}$ but changes in activity patterns that underlie

\footnotetext{
*Address all correspondence to Lan Luan, lan.luan@ @ice.edu

${ }^{\dagger}$ These authors contributed equally to this work.
} 
adaptation, learning, development, and degeneration occur on broader timescales ranging from seconds to years and even decades. ${ }^{2-4}$ Spatially, neural activity involves not only cellular and subcellular structural and functional changes, but also orchestrated activities distributed across multiple brain areas. In addition to neuronal activity, brain functions and dysfunctions also stem from the interaction between other cell types and surrounding vasculature. ${ }^{5,6}$ Resolving these multifaceted activities requires technologies with cross-brain coverage, long-lasting functioning periods, and high spatial and temporal resolutions that match the network in question.? Electrophysiology, one of the "gold standard" tools in detecting neuronal dynamics, affords high temporal resolution detection and direct neural activation, but is limited by its low spatial resolution incapable of resolving fine cellular and subcellular structures. In contrast, optical imaging and modulation techniques offer high spatial resolution ${ }^{8,9}$ and cell type specificity, ${ }^{10}$ and can measure non-neuronal, non-electrical activities. ${ }^{11,12}$ Nevertheless, they are limited by their generally low temporal resolution and insufficient tissue penetration depth. Integrating the electrical and optical measurements in the same brain leverages their complementary strengths, which has become an emerging approach for synchronously observing and controlling brain activities of a specific region. ${ }^{13-18}$

Among various designs of neural electrodes, flexible electrodes ${ }^{15,19,20}$ are uniquely wellsuited for concurrent, closely opposed optical and electrophysiological data collection. The mechanical flexibility allows these electrodes to be implanted beneath a cranial window and bent out of the way at a small radius without breaking or obstructing the optical view. ${ }^{15,16,20}$ Furthermore, and importantly, their mechanical flexibility alleviates mechanical micromotions ${ }^{21}$ and foreign-body responses ${ }^{22-27}$ at the tissue-electrode interface, which enables long-lasting recordings and stability to track individual neurons. ${ }^{15,28-30}$ However, the same mechanical flexibility makes surgical implantation a challenge. Here we use a $1-\mu \mathrm{m}$-thick, ultraflexible nanoelectronic thread (NET) ${ }^{15}$ to provide step-by-step guidance for surgical co-implantation of flexible electrodes and a cranial window in a single procedure. We include not only surgical procedures, but also preparation of ultraflexible electrodes for implantation and attachment of retractable shuttle devices (Fig. 1). To facilitate the adaptation of this method to other flexible electrodes, we use mostly off-the-shelf components, including cover glass as the cranial window, tungsten microwires as the shuttle devices, and polyetheretherketone (PEEK) microtubes as the guiding structures for shuttle devices. The same procedures can also be adapted to implanting other customized cranial windows of different sizes and materials.

(a)


(b)

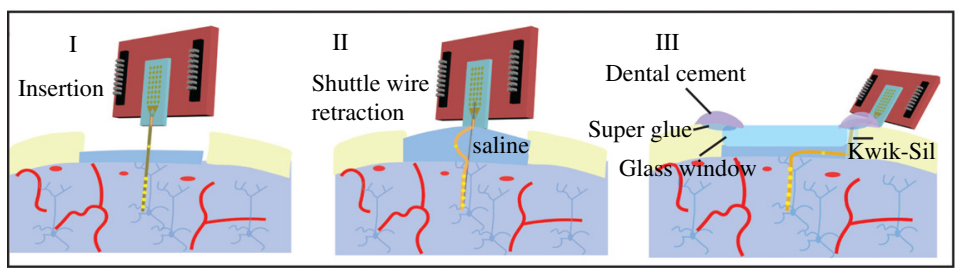

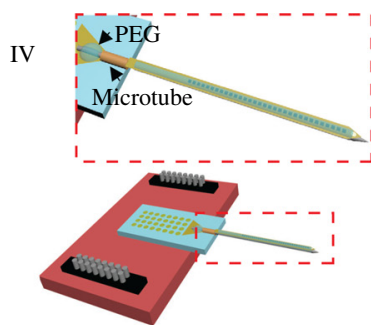

(c)

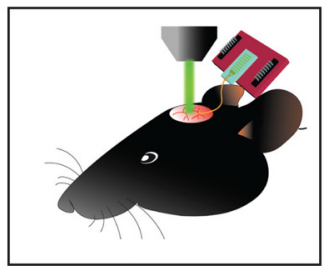

Fig. 1 Schematics showing the overall workflow and outcome. (a) Key steps of NET preparation for implantation, including releasing of the flexible section (I, II) and temporary attachment of a shuttle wire (III, IV). (b) Key steps in cranial surgical procedures, including insertion of NET (I), retraction of the shuttle wire (II), and cranial window installation (III). (c) Post-surgery mouse supporting chronic, multimodal measurements that combine electrophysiology and optical methods. 


\section{Protocol}

This protocol starts with pre-surgery device preparations for which a single-shank 32-channel NET electrode array is temporarily attached to an insertion shuttle made of a tungsten microwire. It proceeds with descriptions of surgical techniques and implantation steps required to successfully implant both the NET array and a cranial window in a mouse (Fig. 1). It ends with representative electrophysiological recordings and optical imaging from the co-implantation of NET and a cranial window.

This protocol assumes the starting materials of a NET array before releasing from the fabrication substrate of glass, a tungsten microwire (diameter: $50 \mu \mathrm{m}$, length: 10 to $15 \mathrm{~mm}$ ) as the insertion shuttle, the bio-dissolvable adhesive polyethylene glycol (PEG) as the temporary adhesive ${ }^{31}$ and a PEEK microtube as the guiding structures for the insertion shuttle (Fig. 2). The prereleased NET array is bonded with a customized printed circuit board (PCB) by a standard ball grid array packaging method, in which solder balls are manually placed on the bonding pad of NETs and brought into contact with the PCB that has matching copper pads. The assembly is then heated in a reflow oven to melt the solder balls and make a permanent bonding. After bonding, epoxy is applied at the edge of the NET device where it meets the PCB as mechanical reinforcement. The tip of the tungsten shuttle is pre-sharpened using $\mathrm{KOH}$ electrochemical etching as previously reported. ${ }^{32}$ This protocol also uses a \#1 cover glass (diameter: $3 \mathrm{~mm}$ ) and a 3D-printed piece to securely attach the NET device to a stereotaxic micromanipulator. All animal-involved protocols described in this protocol have been approved by the Institutional Animal Care and Use Committee at Rice University.

\subsection{Preparation of Ultraflexible NET for Implantation}

\subsubsection{Assembly of PEEK tube and tungsten shuttle}

1. Clean and rinse the NET device in isopropanol, and blow dry.

2. Use a pair of tweezers to attach the PEEK tube near the base of the NET bonding pad [Fig. 3(a)]. The PEEK tube is 6-mm long, extending from the bottom of the bonding pad. Add a small amount of adhesive epoxy on the outside of the tube to fully fix its position and secure it to the NET carrier chip.

3. Retrieve the sharpened tungsten-wire shuttle using a pair of forceps or micro-pincers. Grip and slowly guide the wire shuttle through the PEEK tube. Gently push the wire forward and toward the tip of the NET electrode. Keep driving the shuttle wire through the tube and across the glass surface until reaching the end of the device. The shuttle should stop 300 to $400 \mu \mathrm{m}$ before reaching the tip of the unreleased NET [Figs. 3(a) and 3(b)].

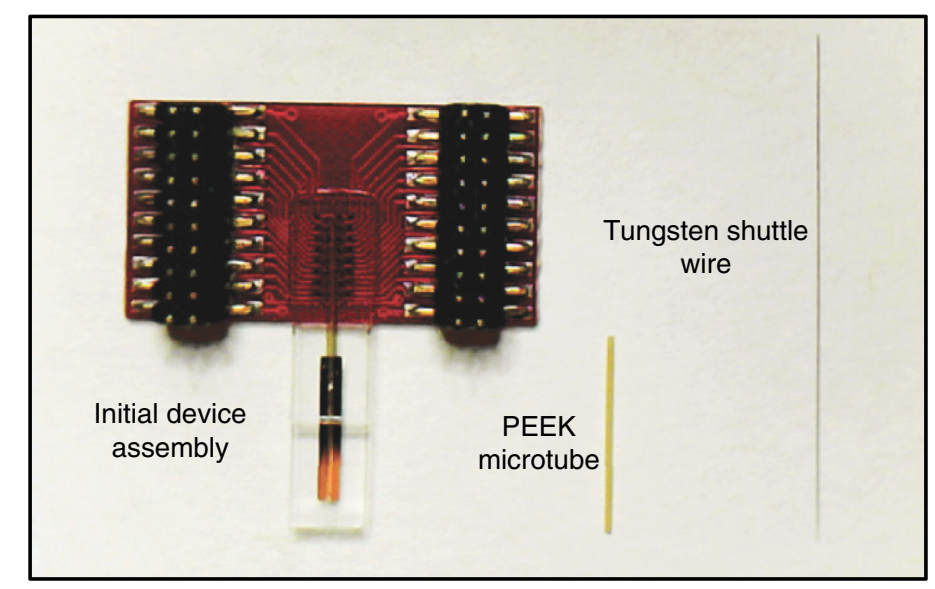

Fig. 2 Insertion shuttle assembly components: an ultraflexible NET device before releasing from the fabricate substrate; a 10 to $15 \mathrm{~mm}$ long $50-\mu \mathrm{m}$ diameter tungsten wire shuttle, and a PEEK capillary tube (O.D. $300 \mu \mathrm{m}$, I. D. $75 \mu \mathrm{m}$ ) for alignment and insertion. 
(a)

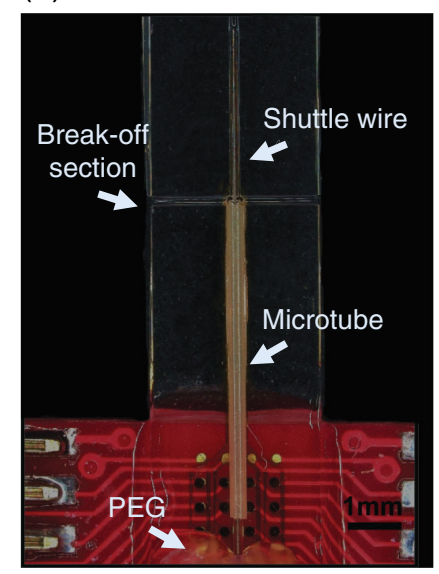

(b)



(c)

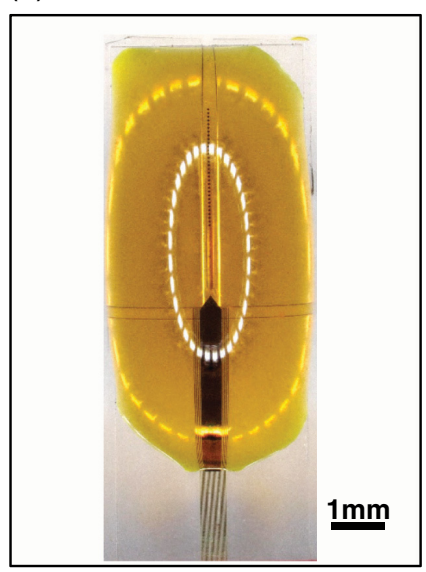

Fig. 3 Procedures of mounting a PEEK tube and a tungsten shuttle, and releasing of NET from the substrate. (a) Front view and (b) side view of the tungsten shuttle feeding through a PEEK tubing. (c) The flexible section of NET immersed in Ni etchant to remove the Ni sacrificial layer.

4. Apply PEG ( $8 \mathrm{k}$ molecular weight) as the dissolvable adhesive between the tungsten microwire and the PEEK tube. This step will fix the tungsten shuttle to its current position, preventing any unintended movements during future handling and insertion.

5. Remove the sacrificial layer $\mathrm{Ni}$ on the NET device to release the flexible section of NET by applying Ni etchant for $\sim 10$ to $30 \mathrm{~min}$ [Fig. 3(c)]. Once all the $\mathrm{Ni}$ is etched away and the device is released, the device will float in the Ni etchant. Wash away any excess $\mathrm{Ni}$ etchant using deionized (DI) water. Keep the released NET section in DI water before attaching it to the shuttle wire.

\subsubsection{Alignment of NET thread to the insertion shuttle}

1. Immerse the released device in DI water. Use a fine needle and carefully place the flexible NET section at least $0.5 \mathrm{~mm}$ away from the cleaving mark on the carrier chip [Fig. 4(a)].

2. Break off the bottom piece of glass by applying concentrated pressure on the cleaving mark using a pair of wafer cleaving/glass breaking pliers [Fig. 4(b)].

3. Manually align the ultraflexible section of NET onto the tungsten wire shuttle [Figs. 4(c) and 4(d)]. Keep the device fixed in place using adhesive tape. Leverage the surface tension of water to wrap the ultraflexible NET onto the tungsten wire. Use a 31-gauge needle to control a single drop of water and the associated water-air surface tension to align the electrode thread on the tungsten shuttle [Fig. 4(e) and supplementary video]. The alignment between the flexible section of NET and the shuttle wire needs to satisfy the following criteria: (a) The individual recording sites on NETs face away from the tungsten wire so they will be in intimate contact with the brain tissue during and after implantation. (b) The tip of the NET thread should not extend beyond the tip of the tungsten wire to decrease the chance of premature detachment during implantation.

4. Once the flexible section of NET and the shuttle wire is appropriately aligned, apply $10 \%$ PEG aqueous solution using a 31-gauge needle to attach NET with the wire. We choose PEG because PEG is biocompatible and biodegradable. The dissolution time by the cerebrospinal fluid is a few minutes, which is conveniently short for prompt implantations, and sufficiently long to support implantations into mouse cortex and hippocampus. Start at the tip of the shuttle device and work along the thread to the end of the flexible section. The thickness of the PEG adhesion layer should not exceed two microns ${ }^{32}$ and therefore not easily visible under the microscope [Fig. 4(d)].

Note: If the flexible device is placed too short or too long from the sharpened end of the shuttle, adjust the wire's position by first dissolving the PEG attaching the shuttle 
(a)

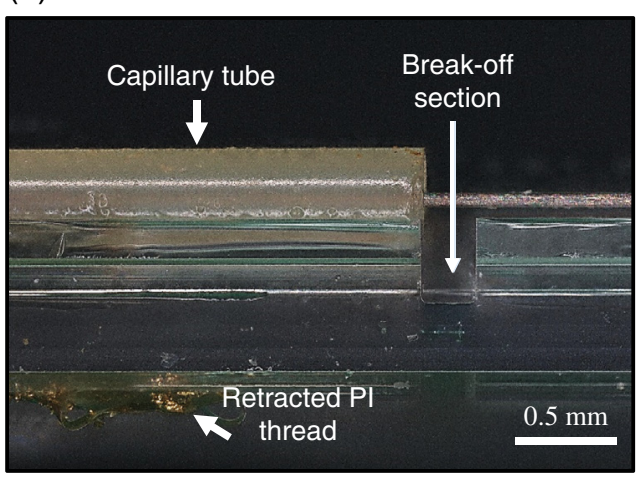

(c)

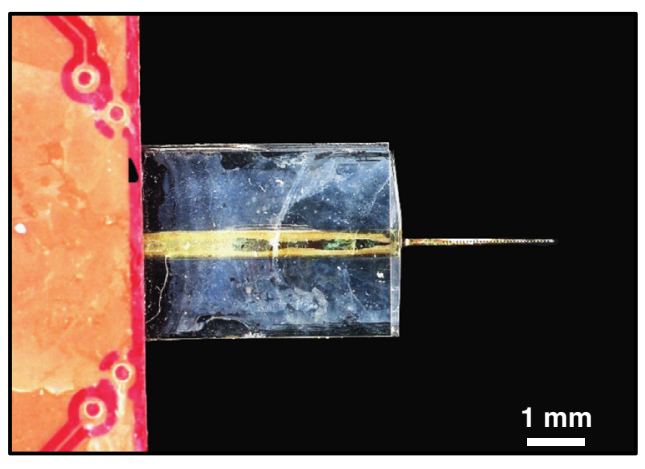

(b)

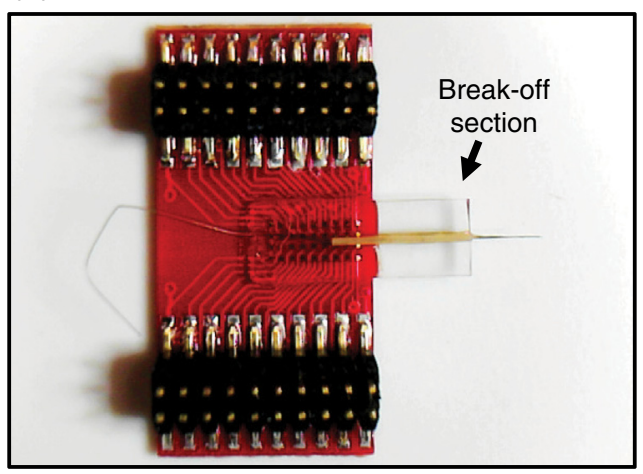

(d)

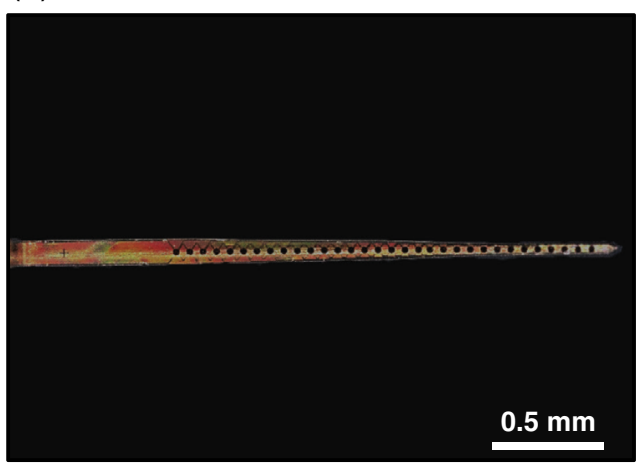

(e)
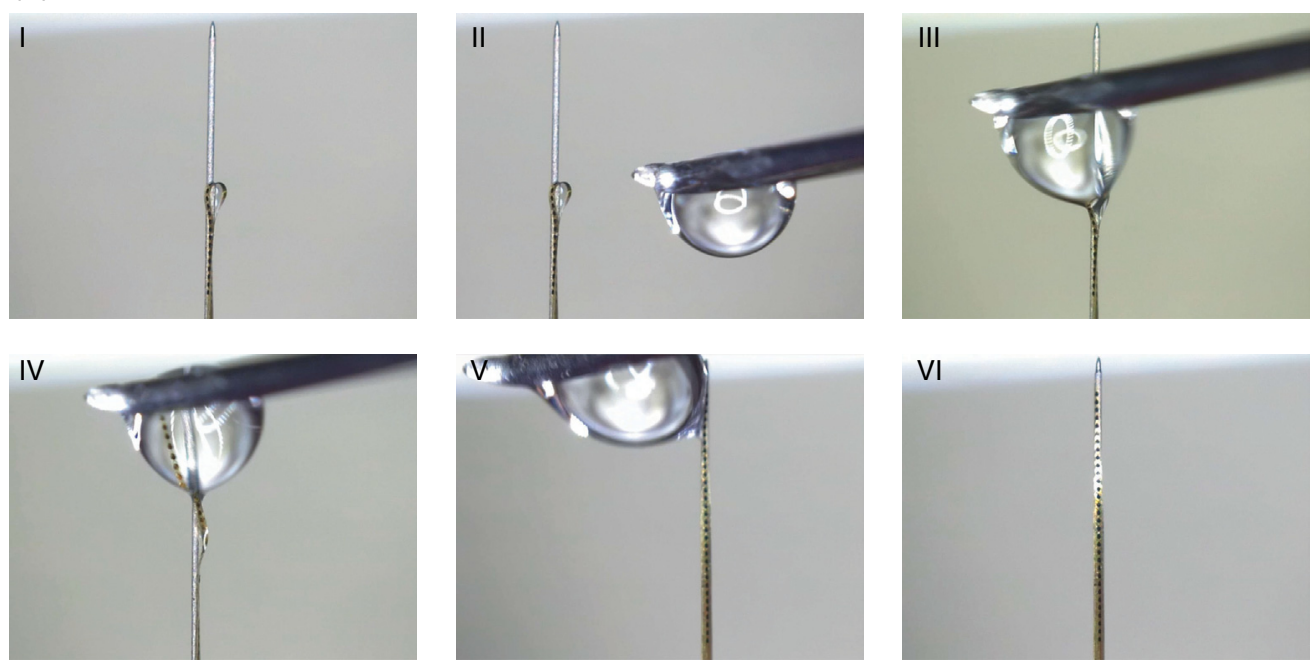

Fig. 4 Procedures of aligning a NET to an insertion shuttle. (a) Zoom-in side view of a NET device showing the relative position of the PEEK tube, the tungsten shuttle, and the retracted flexible section of NET before cleaving off a section of the substrate. (b)-(d) NET-shuttle ensemble overview (b, c) and zoom-in view of NET aligned on the tungsten shuttle. (e) Snapshots showing the NET-shuttle alignment procedures that leveraged the surface tension of a water droplet.

to the PEEK tube. Reposition the shuttle using a pair of tweezers to the proper position and reapply PEG to re-fix its location.

5. Sterilize the assembled device by ethylene oxide low-temperature sterilization. The total weight of the implant is $<1 \mathrm{~g}$. 


\subsection{Surgical Procedures for Co-Implantation of NET and a Cranial Window}

\subsubsection{Craniotomy}

1. Anesthetize the animal using isoflurane (3\% for induction and $1 \%$ to $2 \%$ for maintenance). Place the animal on a homeothermic blanket at $37^{\circ} \mathrm{C}$ to maintain body temperature stable. Monitor the depth of anesthesia by periodic toe-pinch tests (every $15 \mathrm{~min}$ ) and the breath rate (55 to 65 breaths/ $\mathrm{min}$ ).

2. Administer Ethiqa-XR $(3.25 \mathrm{mg} / \mathrm{kg})$ for analgesia and dexamethasone $(2 \mathrm{mg} / \mathrm{kg}$, subcutaneous) for anti-inflammation after induction. Apply ophthalmic ointment on the eyes to prevent drying of the cornea. Administer warmed lactated ringers subcutaneously $1 \mathrm{ml} / 100 \mathrm{~g}$ body weight per anesthesia hour to minimize postoperative dehydration.

3. Shave fur on the surgical site with a clipper.

4. Fix the mouse on the stereotaxic apparatus. Position the intra aural positioning studs to symmetrically fix the position of the animal's head in the apparatus. Use only blunted style ear studs for survival procedures. To insert the incisor adapter, use small forceps to pull down the animal's lower jaw and slowly move the incisor adapter into the mouth until the incisors fit in the opening; gently pull back slightly and fix the adapter in place. Gently position the nose clamp by resting on top of the nose using very low pressure. Adjust the incisor screw to make the head level so that bregma and lambda are equal in height. Level the head horizontally in the caudal-to-rostral direction.

5. Sterilize the surgical site via alternating ( $3 \times)$ scrubbing of iodine (betadine) and $70 \%$ isopropyl alcohol. Administer $0.5 \%$ Lidocaine $(7 \mathrm{mg} / \mathrm{kg})$ underneath the scalp.

6. Incise into the skin of the scalp and resect to expose the skull between bregma and lambda skull sutures [Fig. 5(a)]. Remove the periosteum and score the skull with the tip of a scalpel blade to roughen the skull.

7. Measure the desired coordinates with a pair of calipers and use a scalpel blade to mark the position. Thin the bone along the marked craniotomy perimeter using a surgical drill. Drill a circular craniotomy with a diameter of $3 \mathrm{~mm}$ over the target brain areas [Fig. 5(b)].

Note: Regularly rinse the skull with sterile saline during drilling to prevent heating. The thickness of the bone along the drilled perimeter can be checked by gently pressing on the bone. Drill until the central piece of the bone is detached from the rest of the skull.

8. Using a pair of forceps, carefully lift the central piece of the skull. If dura is attached to some part of the skull, apply sterile saline, slowly pry the edge, and follow around.

9. Immediately after removing the central piece of the skull, place a piece of saline-soaked gel foam in the craniotomy to keep the exposed brain moist and promote coagulation.

10. Carefully remove any remaining thin layer of bone on the edge of the craniotomy. This prevents rapid bone regrowth under the window that impedes optical clarity for chronic studies. Remove small residues of bone dust and blood clots.

11. Drill a burr hole at the contralateral hemisphere of the brain and insert a metal wire (made of platinum, silver, or medical-grade stainless steel) as the grounding reference.

(a)

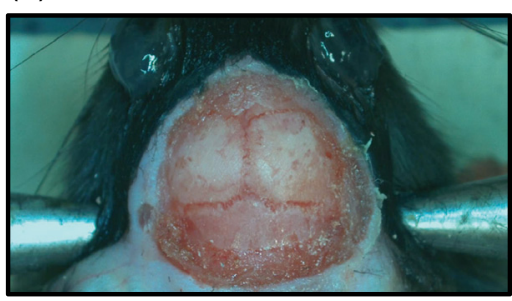

(b)

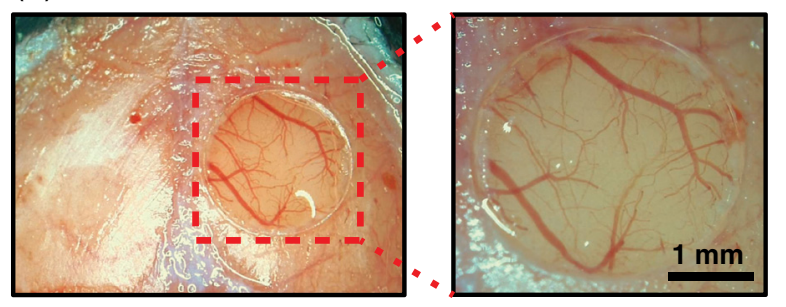

Fig. 5 Preparation of the skull and craniotomy. (a) An animal with scalp removed and skull prepared for craniotomy. (b) A 3-mm diameter cranial window opened above the somatosensory cortex. A zoom-in image shows minimal vessel damage and a clear 3-mm glass window securely fitting within the craniotomy. 


\subsubsection{Implantation of NET}

1. Fix the assembled NET device onto a stereotaxic arm using a 3D-printed case and a pair of screws [Fig. 6(a)].

2. Remove any gel foam from the craniotomy. Aim the device to the insertion site. To avoid surface vasculature, move in z-direction toward the brain and fine adjust the implantation location [Fig. 6(b-I)].

Note: Avoid prolonged periods of the device in close proximity to the brain before insertion. Water vapor and condensation from the brain surface may weaken the adhesion between the NET and the insertion shuttle, leading to premature detachment prior to or during insertion.

3. Insert the device quickly. For a thin layer (1 to $2 \mu \mathrm{m}$ ) of PEG as we demonstrate here, the insertion time should be within $5 \mathrm{~s}$ to avoid pre-mature separation between the shuttle wire and PEG. To achieve slower insertion speed, thicker layer of PEG can be used at the expense of larger implantation footprint. After insertion, dissolve PEG outside the brain by gently dripping body-temperature saline on the device where PEG is adhered to the shuttle needle. Wait until PEG outside the brain is completely dissolved $(<10 \mathrm{~min})$. The device will float in saline and separate from the shuttle needle, indicating it is ready for retraction [Fig. 6(b-II)]. By this time, PEG inside the brain should have also been dissolved by cerebrospinal fluid.

4. Grip the PCB end of the shuttle wire using a pair of tweezers and retract the shuttle wire manually. During retraction, carefully inspect the interface between the implanted NET and the shuttle needle through the surgical microscope. The implanted section of NET should not move with the shuttle wire.

(a)



(b)

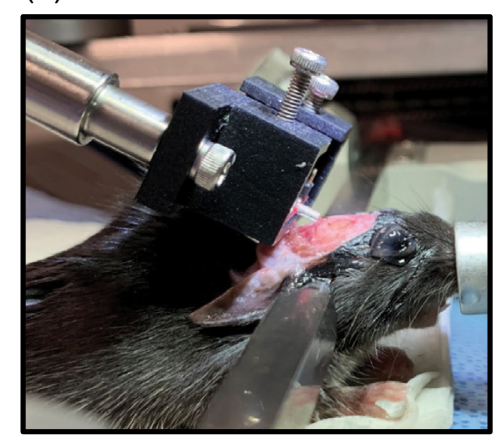

(c)

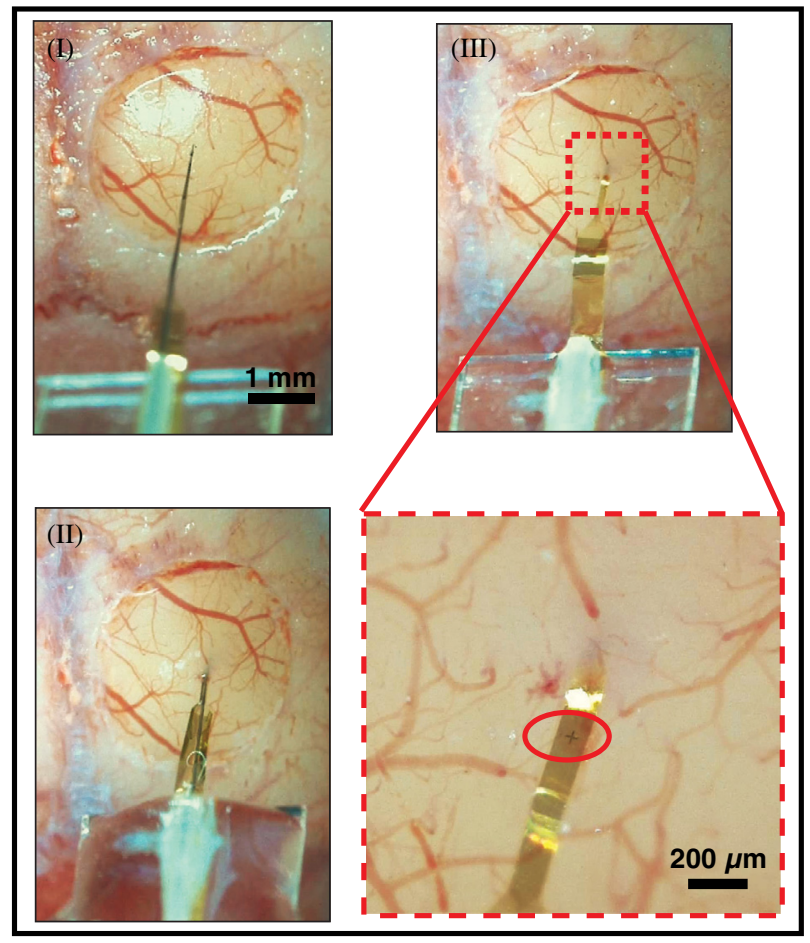

Fig. 6 Intracortical implantation of NETs. (a) A NET device mounted on a stereotaxic arm with a 3D-printed fixation case. (b) Side view showing the mounting angle of the carrier chip to minimize interference with imaging objectives. (c) Implantation of the NET device. (I) Target the brain region of interest and avoid blood vessels. (II) Fast insertion of the NET with the tungsten shuttle. Dissolve PEG with saline to release NET from the shuttle. (III) Retraction of the tungsten shuttle and fine adjustment of implantation depth. Zoom-in image shows the pre-designed "+" marker (red oval) on NET for implantation depth control. 
5. To achieve precise implantation depth after shuttle needle is retracted, slowly retract the stereotaxic arm, straighten the flexible section outside the brain, and pull out the implanted section to reach the designated depth. Depth markers are fabricated on NET as guidance [Fig. 6(b-III)].

6. Rotate the stereotaxic arm to reduce the mounting angle of the carrier chip on the skull. Depending on the rotation axis, this may involve iterative steps of rotation and moving the micromanipulator on the stereotaxic arm. Avoid straightening the flexible section of NET outside the brain to minimize uncontrolled stress on it [Fig. 6(b-III)]. The carrier chip should be more than $2 \mathrm{~mm}$ away from the edge of the craniotomy and at an angle of $45 \mathrm{deg}$ or shallower to accommodate the optical objectives for two-photon imaging [Fig. 6(c)]. The flexible section of NET outside the brain should have some slack (at least $500 \mu \mathrm{m}$ ) to accommodate possible tissue motion as initial implantation damage subsides with time. Fix the stereotaxic arm when the carrier chip reaches the final location and angle.

\subsubsection{Implantation of chronic cranial window}

1. After implantation of NET, add sterile saline to the brain. Place a glass window on the exposed area. If there is space between the window and the remaining skull, fill it with Kwik-sil.

2. Dry the skull. Apply a layer of low viscosity super glue on the remaining skull. Apply a layer of high viscosity super glue around the edge of the window, ensuring contact with the skull.

3. Apply a layer of Metabond dental cement over the high-viscosity super glue and the Kwik-sil, and apply additional cement to fix the NET carrier chip to the skull [Fig. 7(a)].

4. Mount a lightweight titanium headbar on the skull for head-restrained experiment by applying another layer of Metabond cement [Fig. 7(b)].

5. Cap the glass window using a piece of silicone to protect the window from mechanical scratches and tissue from photobleaching by ambient light.

\subsubsection{Postoperative care}

Animals will be monitored and documented twice in the first $24 \mathrm{~h}$ post-surgery, then daily for four days on body weight, incisional appearance, posture and attitude (grimacing, squinting, walking on toes or hunched. Afterward, the mouse status (body weight; body condition, score as defined by Rice University's "Monitoring Metric Scoring for Humane Endpoints to Score Animal Discomfort After Surgery" form; head cap condition) is documented weekly post-surgery.

\subsection{Representative Results}

After this protocol, a 32-channel NET was co-implanted with a glass cranial window over the mouse motor cortex, resulting in closed opposed electrophysiological and optical data collection

(a)

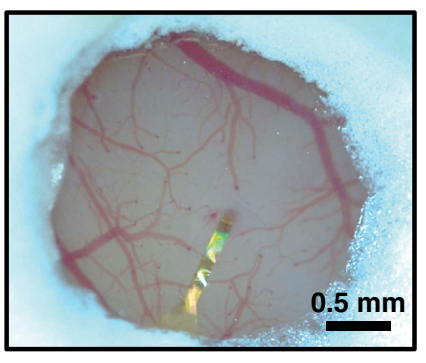

(b)

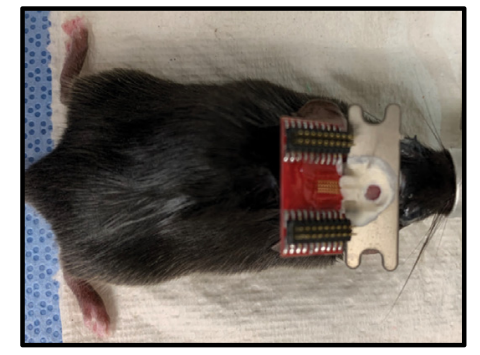

(c)

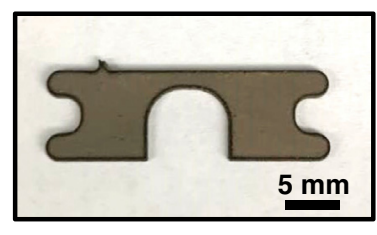

Fig. 7 Co-implantation of the cranial window and a head-plate. (a) Installed cranial window on top of the NET. white shows the Metabond cement. (b) Overview of the headstage after implantation. NET chip carrier is mounted at an oblique angle. (c) The design of the titanium headbar for headfixed, awake experiments. 
(a)
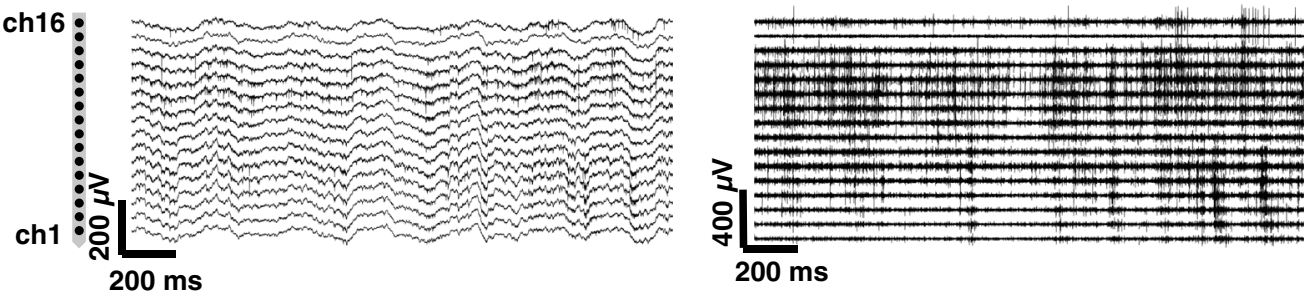

(b)

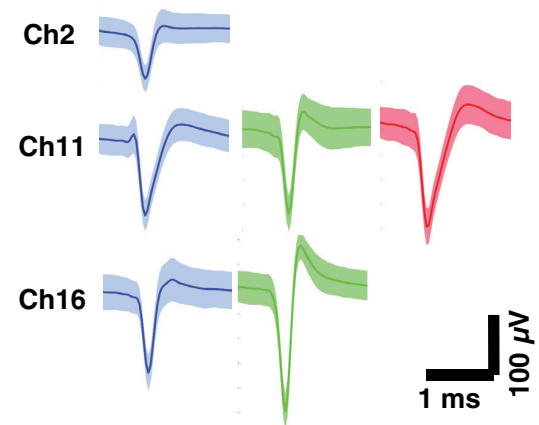

(c)

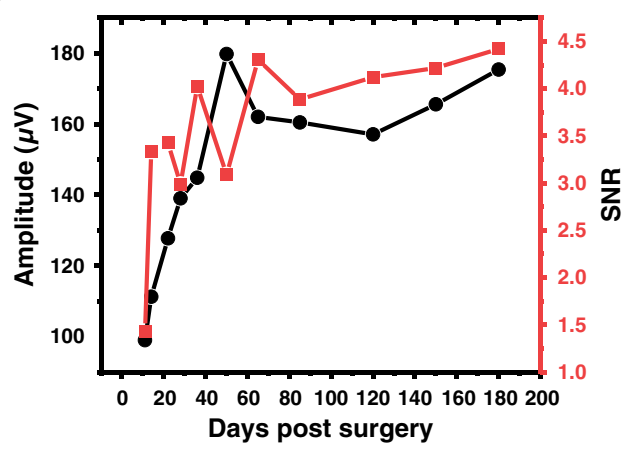

(e)
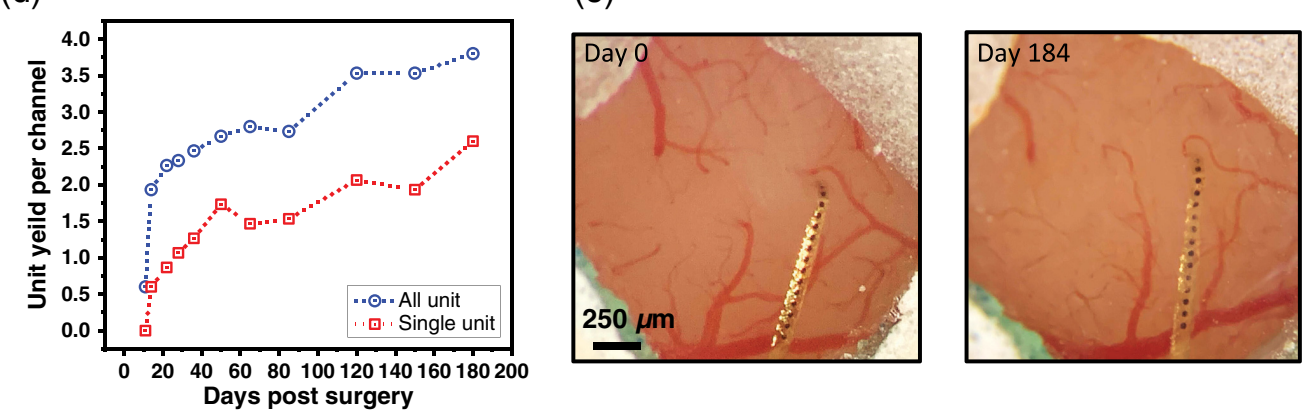

(f)

(g)

(h)
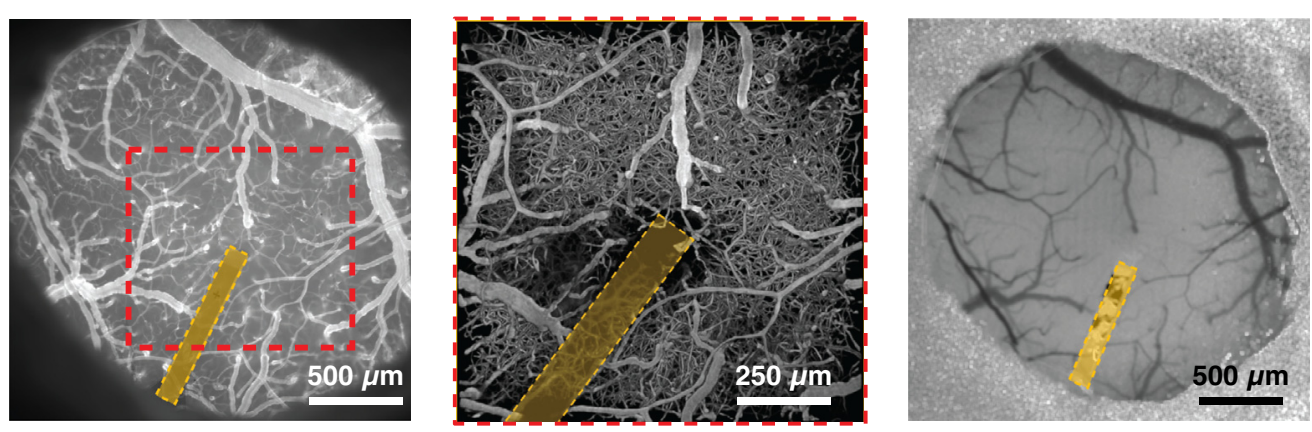

Fig. 8 Representative results of closely opposed electrophysiological recordings and optical imaging of multiple modalities. (a) Unfiltered voltage traces (left) and band-pass $(300-6000 \mathrm{~Hz}$ ) filtered voltage traces (right) from 16 channels spanning the depth of the mouse cortex. (b) Single units isolated from three representative channels in (a). Solid line: average waveform; shade: standard deviation. (c) The averaged amplitude of all units and the averaged SNR of the longitudinal recordings in (a). (d) The all unit yield and single unit yield for all the channels of the longitudinal recordings. (e) Co-implanted cranial window at the day of surgery (day 0 ) and 184 days after surgery showing that the method affords long-lasting optical access. (f) Wide field fluorescence imaging of pial vessels. (g) Two photon imaging of vasculature from the dashed square in (f). Blood plasma was labeled by fluorescein isothiocyanate-dextran (FITC-dextran). Maximum intensity projection of a $600-\mu \mathrm{m}$-thick cortical microvascular stack is shown. (h) Laser speckle contrast imaging of cerebral blood flow surrounding the implanted NET. Orange band denotes NET in (f)-(h). 
that lasted over 180 days. Figures 8(a) and 8(b) shows the representative recordings from 16 channels and representative units detected from the recordings at Day 180 after implantation. The unit yield, averaged amplitude of all units, and the signal-to-noise ratio (SNR) increased and fluctuated in the first 60 days, and remained stable for the rest period without any decay over the time course of 184 days [Figs. 8(c) and 8(d)]. Here "all unit" represents all sorted units from Mountain Sort after rejecting noise and artifact clusters. ${ }^{33}$ "single unit" represents the units that meet the single unit criteria: the number of spikes with an inter-spike interval smaller than $2 \mathrm{~ms}$ is $<2 \%$ of the total events. The optical window remained clear without significant dura or bone regrowth for the same 6 month period [Fig. 8(e)]. In another animal with a co-implanted 32channel NET and a glass cranial window over the somatosensory cortex, we showcased a variety of imaging modalities, including wide-field fluorescence imaging ${ }^{34}$ [Fig. 8(f)] and two-photon imaging of vasculature ${ }^{9}[\mathrm{Fig} .8(\mathrm{~g})]$, and laser speckle contrast imaging of cerebral blood flow ${ }^{35}$ [Fig. 8(h)]. Mice were awake, head-fixed on a custom-made free-moving treadmill for these measurements. These results show the unique capabilities of the method described in this protocol in enabling longitudinal measurements of the multifaceted brain activity.

\section{Discussion}

This protocol provides step-by-step instruction on the implantation preparation of ultraflexible NET and its co-implantation with a cranial window, with the goal of disseminating these reproducible methods to the neuroscience community. While the protocol described here uses a single-shank NET as an example, the same procedures apply to multi-shank flexible electrodes as previously discussed. ${ }^{32}$

The methods use off-the-shelf, cost-effective components such as tungsten microwires and PEG, which is beneficial for their facile adaptation to other designs of flexible electrodes. One caveat associated with this method is the relatively low level of control in the dissolution time of PEG, resulting from the simple design of the shuttle device and adhesion mechanism. A more sophisticated design of the shuttle device, e.g., containing microfabricated reservoirs of PEG, ${ }^{36}$ can alleviate this problem. An alternative shuttle-device attachment mechanism, such as the needle and thread sewing mechanism, ${ }^{15}$ provides a promising approach to precisely control when and where the insertion shuttle detaches from the flexible electrodes.

\section{Materials}

Table 1 lists materials and relevant information.

Table 1 Materials list.

\begin{tabular}{|c|c|c|c|}
\hline Name & Company & Catalog number & Comments \\
\hline PEEK thin-wall tubing & Zeus & B00169K9NW & Guiding microtubes \\
\hline Tungsten wire & $\begin{array}{l}\text { Advent Research } \\
\text { Materials }\end{array}$ & W560604 & Diameter of $75 \mu \mathrm{m}$ \\
\hline Polyethylene glycol & Millipore Sigma & 8188921000 & $\begin{array}{l}\text { Bioadhesive } \\
\text { Molecular weight: } 35000\end{array}$ \\
\hline Nickel Etchant Type I & Transene Company & 012027 & $\begin{array}{l}\text { Nickel Etchant Type I contains metal } \\
\text { chelating ingredients for optimal } \\
\text { performance. Nickel Etchant Type I } \\
\text { will not attack gold films. }\end{array}$ \\
\hline Glass window & Warner Instruments & $64-0720$ & No. 1 cover glass, diameter: $3 \mathrm{~mm}$ \\
\hline Kwik-sil & $\begin{array}{l}\text { World Precision } \\
\text { Instruments }\end{array}$ & NA & $\begin{array}{l}\text { Fill the gap between the skull and } \\
\text { the cranial window }\end{array}$ \\
\hline
\end{tabular}


Table 1 (Continued).

\begin{tabular}{|c|c|c|c|}
\hline Name & Company & Catalog number & Comments \\
\hline Super glue & Loctite & 43903 & $\begin{array}{l}\text { Applied on Kwil-sil to promote the } \\
\text { adhesion of Metabond cement } \\
\text { to the glass window }\end{array}$ \\
\hline Metabond cement & Parkell & s399 & $\begin{array}{l}\text { For sealing the cranial window and } \\
\text { mounting the carrier chip to the skull }\end{array}$ \\
\hline $\begin{array}{l}\text { Kopf stereotaxic } \\
\text { instrument }\end{array}$ & Kopf Instruments & Model 940 & For mice \\
\hline 3D-printed pieces & NA & NA & $\begin{array}{l}\text { Files available at: https://github.com/ } \\
\text { yinrocky/LuanLab3Ddesigns }\end{array}$ \\
\hline
\end{tabular}

\section{Disclosures}

L.L. and C.X. are co-inventors on a patent on the ultraflexible neural electrode technology described herein, filed by The University of Texas (WO2019051163A1, 14 March 2019). L.L. and C.X. hold equity ownership in Neuralthread Inc., an entity that is licensing this technology. The authors declare no other competing interests.

\section{Acknowledgments}

This work was funded by the National Institute of Neurological Disorders and Stroke, under U01 NS115588 (to C.X.), R01NS109361 (to L.L.), and R01NS102917 (to C.X.); and the National Heart, Lung, and Blood Institute, under K25HL140153 (to L.L.).

\section{Code, Data, and Materials Availability}

All data needed to evaluate the conclusions in the paper are present in the paper. Additional data related to this paper may be requested from the authors.

\section{References}

1. A. L. Hodgkin and A. F. Huxley, "A quantitative description of membrane current and its application to conduction and excitation in nerve," J. Physiol. 117, 500-544 (1952).

2. E. L. Bennett et al., "Chemical and anatomical plasticity brain," Science 146, 610-619 (1964).

3. W. J. Freed, L. de Medinaceli, and R. J. Wyatt, "Promoting functional plasticity in the damaged nervous system," Science 227, 1544-1552 (1985).

4. S. N. Burke and C. A. Barnes, "Neural plasticity in the ageing brain," Nat. Rev. Neurosci. 7, 30-40 (2006).

5. H. Girouard and C. Iadecola, "Neurovascular coupling in the normal brain and in hypertension, stroke, and Alzheimer disease," J. Appl. Physiol. 100, 328-335 (2006).

6. S. Schaeffer and C. Iadecola, "Revisiting the neurovascular unit," Nat. Neurosci. 24, 1198-1209 (2021).

7. L. Luan et al., "Recent advances in electrical neural interface engineering: minimal invasiveness, longevity, and scalability," Neuron 108, 302-321 (2020).

8. Y. Adam et al., "Voltage imaging and optogenetics reveal behaviour-dependent changes in hippocampal dynamics," Nature 569, 413-417 (2019).

9. F. Helmchen and W. Denk, "Deep tissue two-photon microscopy," Nat. Methods 2, 932-940 (2005). 
10. E. S. Boyden et al., "Millisecond-timescale, genetically targeted optical control of neural activity," Nat. Neurosci. 8, 1263-1268 (2005).

11. D. Kleinfeld et al., "Fluctuations and stimulus-induced changes in blood flow observed in individual capillaries in layers 2 through 4 of rat neocortex," Proc. Natl. Acad. Sci. U.S.A. 95, 15741-15746 (1998).

12. E. Bindocci et al., "Three-dimensional $\mathrm{Ca}(2+)$ imaging advances understanding of astrocyte biology," Science 356, 8185 (2017).

13. J. Lee et al., "Transparent intracortical microprobe array for simultaneous spatiotemporal optical stimulation and multichannel electrical recording," Nat. Methods 12, 1157-1162 (2015).

14. D. W. Park et al., "Fabrication and utility of a transparent graphene neural electrode array for electrophysiology, in vivo imaging, and optogenetics," Nat. Protoc. 11, 2201-2222 (2016).

15. L. Luan et al., "Ultraflexible nanoelectronic probes form reliable, glial scar-free neural integration," Sci. Adv. 3, e1601966 (2017).

16. L. Luan et al., "Nanoelectronics enabled chronic multimodal neural platform in a mouse ischemic model," J. Neurosci. Methods 295, 68-76 (2018).

17. F. He et al., "Multimodal mapping of neural activity and cerebral blood flow reveals longlasting neurovascular dissociations after small-scale strokes," Sci. Adv. 6, eaba1933 (2020).

18. M. Thunemann et al., "Deep 2-photon imaging and artifact-free optogenetics through transparent graphene microelectrode arrays," Nat. Commun. 9, 2035 (2018).

19. W. Lee et al., "Transparent, conformable, active multielectrode array using organic electrochemical transistors," Proc. Natl. Acad. Sci. U.S.A. 114, 10554-10559 (2017).

20. X. Wei et al., "Nanofabricated ultraflexible electrode arrays for high-density intracortical recording," Adv. Sci. 5, 1700625 (2018).

21. A. Gilletti and J. Muthuswamy, "Brain micromotion around implants in the rodent somatosensory cortex," J. Neural Eng. 3, 189-195 (2006).

22. P. J. Rousche and R. A. Normann, "Chronic recording capability of the Utah Intracortical Electrode Array in cat sensory cortex," J. Neurosci. Methods 82, 1-15 (1998).

23. J. C. Williams, R. L. Rennaker, and D. R. Kipke, "Long-term neural recording characteristics of wire microelectrode arrays implanted in cerebral cortex," Brain Res. Brain Res. Protoc. 4, 303-313 (1999).

24. D. R. Kipke et al., "Silicon-substrate intracortical microelectrode arrays for long-term recording of neuronal spike activity in cerebral cortex," IEEE Trans. Neural Syst. Rehabil. Eng. 11, 151-155 (2003).

25. J. C. Barrese et al., "Failure mode analysis of silicon-based intracortical microelectrode arrays in non-human primates," J. Neural Eng. 10, 066014 (2013).

26. J. D. Simeral et al., "Neural control of cursor trajectory and click by a human with tetraplegia 1000 days after implant of an intracortical microelectrode array," J. Neural Eng. 8, 025027 (2011).

27. G. W. Fraser and A. B. Schwartz, "Recording from the same neurons chronically in motor cortex," J. Neurophysiol. 107, 1970-1978 (2012).

28. C. Xie et al., "Three-dimensional macroporous nanoelectronic networks as minimally invasive brain probes," Nat. Mater. 14, 1286-1292 (2015).

29. T. M. Fu et al., "Stable long-term chronic brain mapping at the single-neuron level," Nat. Methods 13, 875-882 (2016).

30. J. E. Chung et al., "High-density, long-lasting, and multi-region electrophysiological recordings using polymer electrode arrays," Neuron 101, 21-31.e5 (2019).

31. S. H. Felix et al., "Insertion of flexible neural probes using rigid stiffeners attached with biodissolvable adhesive," J. Vis. Exp. (79), e50609 (2013).

32. Z. Zhao et al., "Parallel, minimally-invasive implantation of ultra-flexible neural electrode arrays," J. Neural Eng. 16, 035001 (2019).

33. J. E. Chung et al., "A fully automated approach to spike sorting," Neuron 95, 1381-1394.e6 (2017).

34. J. A. Cardin, M. C. Crair, and M. J. Higley, "Mesoscopic imaging: shining a wide light on large-scale neural dynamics," Neuron 108, 33-43 (2020). 
35. A. K. Dunn, "Laser speckle contrast imaging of cerebral blood flow," Ann. Biomed. Eng. 40, 367-377 (2012).

36. H. R. Joo et al., "A microfabricated, 3D-sharpened silicon shuttle for insertion of flexible electrode arrays through dura mater into brain," J. Neural Eng. 16, 066021 (2019).

Rongkang Yin received his $\mathrm{PhD}$ in integrated life science from Peking University in 2019. Currently, he is a postdoctoral fellow working in the Luan Laboratory of Integrative Neural Interface at Rice University.

Brian C. Noble received his BS degree in physics from the University of North Florida in 2018. $\mathrm{He}$ currently is a PhD student in the applied physics program at Rice University working in the Luan Laboratory of Integrative Neural Interface.

Fei He received his $\mathrm{PhD}$ in optical engineering from Zhejiang University in 2010. He worked as a postdoctoral fellow in the Luan Laboratory of Integrative Neural Interface from 2017 to 2021. He is now a principal investigator at Shanghai Institute of Optics and Fine Mechanics, Chinese Academy of Sciences, working on the interdisciplinary brain sciences and optoelectronics.

Palvo Zolotavin received his PhD in physical chemistry from the University of Chicago in 2013. After graduation he became a postdoctoral research associate at the Department of Physics at Rice University. He then joined Lam Research as a process engineer. He is currently a research scientist in Professor Chong Xie's Nanoscale Neural Interface Laboratory at Rice University.

Haad Rathore received his BS in electrical engineering from Lahore University of Management Science in 2017 and his master's degree in applied physics from Rice University in 2019. He is currently a PhD student in the Applied Physics Program at Rice University working in the Luan Laboratory of Integrative Neural Interface.

Yifu Jin received his BS in bioengineering from University of California San Diego in 2019. $\mathrm{He}$ is now a PhD student in the Department of Electrical and Computer Engineering at Rice University working in the Luan Laboratory of Integrative Neural Interface.

Nicole Sevilla received her BS degree in biomedical engineering from Florida International University in 2019. She is currently a PhD student in the Department of Bioengineering at Rice University working in the Luan Laboratory of Integrative Neural Interface

Chong Xie is currently an associate professor of electrical and computer engineering and of bioengineering at Rice University, and a core faculty member of the Rice Neuroengineering Initiative. His research focuses on the development of ultraflexible neural electrodes for largescale, long-lasting neural interfaces.

Lan Luan is currently an assistant professor of electrical and computer engineering and of bioengineering at Rice University, and a core faculty member of the Rice Neuroengineering Initiative. Her laboratory works on the development of multimodal neural interface integrating optical and electrophysiological measurements and their application in neurological disorders. 